\title{
ELOVL2 promotes cancer progression by inhibiting cell apoptosis in renal cell carcinoma
}

\author{
KEN TANAKA ${ }^{1}$, SHUYA KANDORI ${ }^{1}$, SHOTARO SAKKA ${ }^{1}$, SATOSHI NITTA $^{1}$, KOZABURO TANUMA $^{1}$, \\ MASANOBU SHIGA ${ }^{1}$, YOSHIYUKI NAGUMO ${ }^{1}$, HIROMITSU NEGORO ${ }^{1}$, TAKAHIRO KOJIMA ${ }^{1}$, \\ BRYAN J. MATHIS ${ }^{2}$, TORU SHIMAZUI ${ }^{3}$, MAKOTO WATANABE ${ }^{4}$, TAKA-AKI SATO ${ }^{4}$, \\ TAKAFUMI MIYAMOTO ${ }^{5}$, TAKASHI MATSUZAKA ${ }^{5}$, HITOSHI SHIMANO ${ }^{5}$ and HIROYUKI NISHIYAMA ${ }^{1}$ \\ ${ }^{1}$ Department of Urology, Faculty of Medicine, University of Tsukuba, Tsukuba, Ibaraki 305-8577; \\ ${ }^{2}$ International Medical Center, University of Tsukuba Affiliated Hospital, Tsukuba, Ibaraki 305-8576; \\ ${ }^{3}$ Department of Urology, Ibaraki Prefectural Central Hospital, Kasama, Ibaraki 309-1793; \\ ${ }^{4}$ Life Science Research Center, Technology Research Laboratory, Shimadzu Corporation, \\ Kyoto 604-8511; ${ }^{5}$ Department of Endocrinology and Metabolism, Faculty of Medicine, \\ University of Tsukuba, Tsukuba, Ibaraki 305-8575, Japan
}

Received May 27, 2021; Accepted October 27, 2021

DOI: $10.3892 / o r .2021 .8234$

\begin{abstract}
Renal cell carcinoma (RCC) is an aggressive genitourinary malignancy which has been associated with a poor prognosis, particularly in patients with metastasis, its major subtypes being clear cell RCC (ccRCC), papillary PCC (pRCC) and chromophobe RCC (chRCC). The presence of intracellular lipid droplets (LDs) is considered to be a hallmark of ccRCC. The importance of an altered lipid metabolism in ccRCC has been widely recognized. The elongation of very-long-chain fatty acid (ELOVL) catalyzes the elongation of fatty acids (FAs), modulating lipid composition, and is required for normal bodily functions. However, the involvement of elongases in RCC remains unclear. In the present study, the expression of ELOVL2 in ccRCC was examined; in particular, high levels of seven ELOVL isozymes were observed in primary tumors. Of note, elevated ELOVL2 expression levels were observed in ccRCC, as well as in pRCC and chRCC. Furthermore, a higher level of ELOVL2 was significantly associated with the increased incidence of a poor prognosis of patients with ccRCC and pRCC. The CRISPR/Cas9-mediated knockdown of ELOVL2 resulted in the suppression of the elongation of long-chain polyunsaturated FAs and increased LD production in renal cancer cells. Moreover, ELOVL2 ablation resulted in the suppression of
\end{abstract}

Correspondence to: Dr Shuya Kandori, Department of Urology, Faculty of Medicine, University of Tsukuba, 1-1-1 Tennodai, Tsukuba, Ibaraki 305-8577, Japan

E-mail: shuya79@md.tsukuba.ac.jp

Key words: renal cell carcinoma, fatty acid, elongation of very-long-chain fatty acid 2, lipid droplet, cellular proliferation, apoptosis cellular proliferation via the induction of apoptosis in vitro and the attenuation of tumor growth in vivo. On the whole, the present study provides new insight into the tumor proliferation mechanisms involving lipid metabolism, and suggests that ELOVL2 may be an attractive novel target for RCC therapy.

\section{Introduction}

Renal cell carcinoma (RCC) is a commonly encountered and lethal malignancy, accounting for $\sim 2 \%$ of all cancer cases and related deaths worldwide (1), its major subtypes being clear cell RCC (ccRCC), papillary PCC (pRCC) and chromophobe RCC (chRCC). Of all the RCC subtypes, ccRCC is the most common histological manifestation and its pathogenesis is characterized by the constitutive activation of hypoxia-inducible factors (HIFs) due to the loss-of-function in the von Hippel-Lindau (VHL) tumor suppressor gene (1). Various targeted therapies against vascular endothelial growth factor (VEGF) or mammalian target of rapamycin (mTOR) signaling and immune check point inhibitors for metastatic disease have been developed. However, disease progression is inevitable in the majority of patients $(1,2)$.

A hallmark of ccRCC is the abundance of intracellular lipid droplets (LDs), consisting of a neutral lipid core containing triglycerides (TGs) and cholesterol-esters (CEs) surrounded by a phospholipid monolayer (3). LDs are dynamic organelles responsible for lipid uptake and storage, and are used to maintain homeostasis, facilitate energy production and protect against various types of stress or to sustain membrane biogenesis during rapid tumor cell growth in several types of cancer (4). In fact, previous studies have demonstrated that LDs contribute to ccRCC progression $(5,6)$.

The fatty acids (FAs) which comprise the main component of lipids have been reported to serve as substrates for energy storage, membrane synthesis and the production of signaling molecules. Elongation and desaturation are central steps of 
the de novo synthesis of long-chain FAs (LC-FAs), the length and degree of unsaturation being determinants of FA function and metabolic fate (7). Stearoyl-CoA desaturase 1 (SCD1), a member of the fatty acyl desaturase family, has been extensively studied in ccRCC $(6,8,9)$. It has been demonstrated that increased SCD1 expression supports viability, while a SCD1 small molecule inhibitor (A939572) has been shown to suppress cellular proliferation in ccRCC $(8,9)$. Moreover, Yang et al $(10)$ demonstrated that sterol regulatory element-binding protein 1 (SREBP1) promoted lipid desaturation through FA acid desaturase 1 (FADS1) prior to the activation of NF- $\kappa$ B signaling for the promotion of cellular proliferation in ccRCC. However, any potential roles of elongases in RCC remain unclear.

It has been reported that, in mammals, the initial and rate-controlling FA condensation reactions are catalyzed by a family of elongase enzymes referred to as elongation of very-long-chain FAs (ELOVL). To date, seven ELOVL members have been identified, which can be generally divided into those specific for saturated and monounsaturated FAs (ELOVL1, ELOVL3, ELOVL6 and ELOVL7) or for polyunsaturated FAs (PUFAs; ELOVL2, ELOVL4 and ELOVL5). Lucarelli et al (9) revealed a significant accumulation of PUFAs and an increased expression of ELOVL2 and ELOVL5 in ccRCC. Of note, it has been demonstrated that systemic docosahexaenoic acid (DHA) is endogenously produced and controls de novo lipogenesis, while also regulating lipid storage in a sterol regulatory element-binding transcription factor 1 (SREBPF1)-independent manner, due to ELOVL2 ablation in mice (11). Moreover, ELOVL2 overexpression has been shown to promote LD accumulation with an enhanced FA uptake in both the preadipocyte cell line 3T3-L1 and F442A cells (12). Previous in vitro studies, with DHA exogenously administered for cancer cells, have demonstrated that DHA exerts anti-tumorigenic, anti-inflammatory and pro-apoptotic effects on cancer cells (13-15). However, any potential role of ELOVL2 in ccRCC progression through the modulation of lipid metabolism remains largely unexplored.

In the present study, the roles of ELOVL2 in ccRCC progression were explored by performing the transcriptional profiling of primary ccRCC and normal kidney samples, revealing that ELOVL2 is overexpressed in ccRCC and is overrepresented among ELOVL isozymes. Of note, ELOVL2 was also overexpressed in ccRCC, as well as in pRCC and chRCC, this being significantly associated with a poor prognosis of patients with ccRCC or pRCC. Moreover, it was demonstrated that ELOVL2 inhibition affects lipid metabolism and suppresses cellular proliferation via the promotion of apoptosis, at least in part through the disruption of endoplasmic reticulum (ER) homeostasis in renal cancer cells. The results of the present study suggested that ELOVL2 may be a potential novel therapeutic target for RCC treatment.

\section{Materials and methods}

Cell lines and cultures. 293T (RCB2202) and OS-RC-2 (RCB0735) cell lines were purchased from RIKEN BioResource Center while 786-O and ACHN cell lines were purchased from ATCC; SK-RC-52 cells were a kind gift from Dr J.G. Old (Memorial Sloan Kettering Cancer Center). RPTEC cell line, which is derived from epithelial cells of the human renal proximal tubule, was purchased from Lonza Group, Ltd. (CC-2553). The ACHN, 786-O, SK-RC-52 and OS-RC-2 cells were cultured in RPMI-1640 medium supplemented with $10 \%$ fetal bovine serum (FBS) at $37^{\circ} \mathrm{C}$ with a $5 \%$ humidified $\mathrm{CO}_{2}$ atmosphere. The $293 \mathrm{~T}$ cells were cultured in Dulbecco's modified Eagle's medium supplemented with $10 \%$ fetal bovine serum (FBS) at $37^{\circ} \mathrm{C}$ with a $5 \%$ humidified $\mathrm{CO}_{2}$ atmosphere. The RPTEC cells were cultured in renal epithelial growth medium (REGM ${ }^{\mathrm{TM}}$ ) Bulletkit ${ }^{\mathrm{TM}}$ (CC-3190; Lonza Bioscience) at $37^{\circ} \mathrm{C}$ with a $5 \%$ humidified $\mathrm{CO}_{2}$ atmosphere.

Patients and ccRCC samples. RCC tissues and adjacent normal kidney tissues from 46 patients who received radical or partial nephrectomies were obtained from the University of Tsukuba Hospital, between 2006 and 2015 according to the protocols approved by the Ethics Committee of the University of Tsukuba (approval no. H28-104). All patients provided written informed consent prior to undergoing surgery. Tumor stages were assigned according to the TNM staging of the Union for International Cancer Control (16). Pathological grades were classified, according to the four-tiered Fuhrman grading system (17). All patient characteristics are summarized in Table SI.

RNA extraction and cDNA synthesis. Total RNA was extracted from the frozen tissues and renal cancer cells using TRIzol ${ }^{\circledR}$ Reagent (Thermo Fisher Scientific, Inc.). Following RNA purification, RNA was then reverse transcribed into cDNA using a High-Capacity cDNA Reverse Transcription kit (cat. no. 4368814; Thermo Fisher Scientific, Inc.), according to the manufacturer's instructions.

Reverse transcription-quantitative polymerase chain reaction $(R T-q P C R)$. Gene expression levels were quantified using a 7500 Fast Real-Time PCR machine with Fast SYBR-Green Master Mix (Thermo Fisher Scientific, Inc.). The cycling conditions were as follows: Initial hold at $95^{\circ} \mathrm{C}$ for $20 \mathrm{sec}$, 40 cycles at $95^{\circ} \mathrm{C}$ for $3 \mathrm{sec}$ and $60^{\circ} \mathrm{C}$ for $30 \mathrm{sec}$, followed by a melting curve ranging from $95^{\circ} \mathrm{C}$ for $15 \mathrm{sec}$ to $60^{\circ} \mathrm{C}$ for 1 min. Hypoxanthine phosphoribosyltransferase 1 (HPRT1) was used as an internal control. All primer sequences are listed in Table SII. In order to evaluate apoptosis-related genes, the relative expression levels of the pro-apoptotic genes, Bcl-2-associated $\mathrm{X}$ protein (BAX), Bcl-2 homologous antagonist/killer (BAK), phorbol-12-myristate-13-acetate-induced protein 1 (PMAIP1/NOXA) and p53 upregulated modulator of apoptosis (BBC3/PUMA) and of the anti-apoptotic gene BCL2 and induced myeloid leukemia cell differentiation protein gene (MCL1) were analyzed. The relative gene expression levels were quantified using the $2^{-\Delta \Delta \mathrm{Cq}}$ method (18).

Western blot analysis. Western blot analysis was carried out as previously described (19). Cells were lysed in lysis buffer (20 mM Tris- $\mathrm{HCl}$ (pH 7.5), $150 \mathrm{mM} \mathrm{NaCl,} 1 \mathrm{mM}$ EDTA, $1 \%$ SDS and protease inhibitor) and sonicated on ice. The lysates were centrifuged at $1,000 \mathrm{x}$ g for $20 \mathrm{~min}$ at $4^{\circ} \mathrm{C}$ and the supernatants were collected as samples. The protein quantification of samples was performed using Quick Start Bradford Protein assay (cat. no. 5000202JA; Bio-Rad Laboratories, Inc.). Samples were subjected to $10 \%$ SDS-PAGE and separated products were subsequently transferred to PVDF membranes. 
The membranes were blocked with ECL Prime blocking agent (cat. no. RPN418V; Cytiva) for $60 \mathrm{~min}$ at room temperature. The membranes were then incubated overnight at $4^{\circ} \mathrm{C}$ with the following antibodies: Anti-serine/threonine-protein kinase/endoribonuclease inositol-requiring enzyme $1 \alpha$ (anti-IRE1 $\alpha$; 1:200; \#3294, Cell Signaling Technology, Inc.), anti-phosphorylated IRE1 $\alpha$ (anti-p-IRE1 $\alpha ; 1: 200$; NB100-2323, Novus Biologicals, LCC), anti-C/EBP homologous protein (anti-CHOP; 1:200; MA1-250, Thermo Fisher Scientific, Inc.). Anti-rabbit or anti-mouse immunoglobulin G HRP-linked, whole donkey Ab (cat. no. NA934V, NA931VS; GE Healthcare; Cytiva) were used at 1:10,000 as secondary antibodies. The western blots were visualized with ImmunoStar ${ }^{\circledR}$ Zeta (FUJIFILM Wako Pure Chemical Corporation) using a Fujifilm LAS-4000 imager and LAS400IR (FUJIFILM Wako Pure Chemical Corporation). $\beta$-actin was used as the internal control (1:10,000, cat. no. A5316; Sigma-Aldrich).

Bioinformatics analysis of gene expression. Clinical and RNA-sequencing (RNA-seq) data of primary tumors collected from patients with RCC in The Cancer Genome Atlas (TCGA) database were downloaded from the Genomic Data Commons (GDC) Data Portal (20). A total of 1,005 (880 diseased and 125 control) samples were examined, including 530 diseased and 71 control samples for the kidney renal clear cell carcinoma cohort (KIRC; ccRCC), 286 diseased and 31 healthy samples for the cervical kidney renal papillary cell carcinoma cohort (KIRP; pRCC), and 64 diseased and 23 healthy samples for the kidney chromophobe cohort (KICH; chRCC).

ELOVL2 gene expression in the genitourinary cancer samples was analyzed using the Gene Expression database of Normal and Tumor tissues (GENT2). Gene expression data were downloaded from the gene expression omnibus (GEO) public repository of the U133 Plus 2 (GPL570) platform (http://gent2.appex.kr/gent2/) (21).

Plasmids and lentiviral transduction. Small hairpin RNAs (shRNAs) for ELOVL2 characterized in previous studies $(22,23)$ were subcloned into the lentiviral vector pLKO.1 (plasmid \#8453; Addgene, Inc.). The oligonucleotide sequences used in the construction of the shRNA vector are listed in Table SIII. Lentiviruses were generated in 293T cells by co-transfecting four plasmids in trans, including the lentiviral vector (pLKO-shControl or pLKO-shELOVL2), pMDLg/pRRE (plasmid \#12251; Addgene, Inc.), pRSV-Rev (plasmid \#12253; Addgene, Inc.), and pMD2.G (plasmid \#12259; Addgene, Inc.) using Lipofectamine $2000^{\circledR}$ transfection reagent (Thermo Fisher Scientific, Inc.). At $48 \mathrm{~h}$ post-transfection, virus-containing supernatants were collected and filtered through a $0.45-\mu \mathrm{m}$ filter for infection. For viral transductions, pLKO-shControl or pLKO-shELOVL2 lentiviruses were incubated with the 786-O, ACHN and SK-RC-52 cells overnight at $37^{\circ} \mathrm{C}$ in a humidified cell culture incubator. Cells were selected in the presence of puromycin at $24 \mathrm{~h}$ post-infection. To stabilize subclones, cells were cultured in medium with puromycin for 1 month at $37^{\circ} \mathrm{C}$ in a humidified cell culture incubator and surviving pools were utilized for express ion and proliferation analyses.

For overexpression experiments, the OSRC-2 cells were transfected with pCMV6 empty vector (plasmid \#PS100001; Origene
Technologies, Inc.) or pCMV6-ELOVL2 (plasmid \#RC209232; Origene Technologies, Inc.) at $37^{\circ} \mathrm{C}$ in a humidified cell culture incubator, using Lipofectamine $3000^{\circledR}$ transfection reagent (Thermo Fisher Scientific, Inc.), according to the manufacturer's instructions. The cells were used for the indicated assays at $48 \mathrm{~h}$ post-transfection.

CRISPR/Cas9 design and cloning. The pX330-U6-Chimeric_BB-CBh-hSpCas9 (pX330) plasmid was a gift from Feng Zhang (Addgene, Inc.; plasmid \#42230) (24). CRISPR single-guide (sg) sequences for ELOVL2 were specifically targeted to exon 2 (sgELOVL2 \#1) and exon 3 (sgELOVL2 \#2 and \#3) of ELOVL2 using the CRISPR Design Tool (GE Healthcare Dharmacon, Inc.; http://dharmacon.horizondiscovery.com/gene-editing/crispr-cas9/crispr-design-tool/), prior to cloning into $\mathrm{pX} 330$. The CRISPR guide sequence for single-guide control (sgControl) was previously designed (25). The oligonucleotide sequences of the single-guide RNAs (sgRNAs) are listed in Table SIII.

The ACHN cells were then seeded into 6-well plates $\left(1.4 \times 10^{5}\right.$ cells/well) and co-transfected $2 \mathrm{~h}$ later with $2 \mu \mathrm{g}$ of pX330-expresed sgRNAs and $0.2 \mu \mathrm{g}$ pCI-neo vector (Promega Corporation; cat. no. E1841) at $37^{\circ} \mathrm{C}$ in a humidified cell culture incubator, using FuGENE HD (Promega Corporation; cat. no. E2312). At $24 \mathrm{~h}$ post-transfection, $400 \mu \mathrm{g} / \mathrm{ml} \mathrm{G} 418$ were applied for 7 to 10 days and the cells were allowed to recover for 2 or 3 days. When the cells were approaching confluency, they were reseeded sparsely in $10 \mathrm{~cm}$ dishes. After 2 or 3 weeks, discernible colonies were isolated by using cloning discs (MilliporeSigma; cat. no. Z374431-100EA). Individual clones were expanded and evaluated for knockout status by Sanger sequencing for the target area.

Cellular proliferation assays. Cellular proliferation was assessed using MTT assay with a Cell Counting Kit-8 (CCK-8; Dojindo Molecular Technologies, Inc.) according to the manufacturer's instructions. Briefly, the cells were seeded into 96-well plates and $10 \mu \mathrm{l}$ of WST-8 were added after $72 \mathrm{~h}$. OD460 was measured following a $1-\mathrm{h}$ incubation at $37^{\circ} \mathrm{C}$.

Subcutaneous xenografting. BALB/c nude (nu/nu) female mice ( $n=12 ; 6-8$ weeks old) were purchased from Charles River Laboratories, Inc.. The mice were housed under specific pathogen-free conditions, under a 12-h light/dark cycle, with ad libitum access to food and water. For subcutaneous xenograft assays, $1 \times 10^{7}$ cells suspended in $100 \mu 1 \mathrm{PBS}$ were injected subcutaneously into the right flank by using a $24 \mathrm{G}$ needle and the tumor volumes were measured once a week. Tumor volume was calculated by the formula: $\mathrm{mm}^{3}=$ length $\mathrm{x}$ widt h x height x 0.52 (26). After 90 days, all animals were sacrificed, and the xenograft tumors were excised. Animals were anesthetized with $2 \%$ isoflurane before being euthanized by cervical dislocation.

Formalin-fixed and paraffin-embedded (FFPE) specimens of the xenograft tumors were cut into $4-\mu \mathrm{m}$-thick sections, prior to deparaffinization and rehydration. For antigen retrieval, the sections were pretreated by microwave for $21 \mathrm{~min}$ in a citric acid buffer. After the antigen retrieval procedure, endogenous peroxidase activity was blocked with $3 \% \mathrm{H}_{2} \mathrm{O}_{2}$ for $25 \mathrm{~min}$ and the slides were incubated with $\mathrm{Ki}-67$ 
antibody (1:200; Dako; Agilent Technologies, Inc.; M7240) at $4^{\circ} \mathrm{C}$ overnight. The immunohistochemical reaction was visualized using the secondary antibody Histofine Simple Stain MAX PO ${ }^{\circledR}$ (Nichirei Biosciences, Inc,; cat. no. 424151) by using diaminobenzidine as the chromogen.

All animal studies were approved by the Animal Experiment Committee of the University of Tsukuba and all experiments were performed in accordance with the guidelines of the University of Tsukuba's Regulations of Animal Experiments (approval no. 20-370).

Apoptosis assays. Apoptosis was assessed using the Caspase-Glo ${ }^{\circledR}$ 3/7 Assay System (Promega Corporation; cat. no. G8090), Annexin-V-FLUOS staining kit (Roche Diagnostics; cat. no. 11858777001), and JC-1 Mitochondrial Membrane Potential Assay kit (Cayman Chemical Company; cat. no. 10009172), according to the manufacturer's instructions.

Staining of LDs. Cells were seeded on glass coverslips in $60 \mathrm{~mm}$ dishes and cultured with oleic acid $(200 \mu \mathrm{M})$-containing medium at $37^{\circ} \mathrm{C}$ overnight in a $5 \% \mathrm{CO}_{2}$ incubator. The medium was then aspirated, and the cells were washed twice with PBS before fixation with $4 \%$ formaldehyde (FUJIFILM Wako Pure Chemical Corporation) for $5 \mathrm{~min}$ at room temperature. The cells were then washed twice with PBS, and incubated with $0.5 \mu \mathrm{M}$ Lipi-Green (Dojindo Molecular Technologies, Inc.) for $30 \mathrm{~min}$ in the dark at $37^{\circ} \mathrm{C}$. Thereafter, the cells were washed twice with PBS and the coverslips were mounted on glass slides using VECTASHIELD ${ }^{\circledR}$ Antifade Mounting Medium with DAPI (Vector Laboratories, Inc.; cat. no. H-1200). Images of the stained cells were acquired with a BZ-X710 fluorescence microscope (Keyence Corporation).

Live cells were incubated with $0.5 \mu \mathrm{M}$ Lipi-Green in Hanks' Balanced Salt Solution (HBSS) for 30 min before washing twice in HBSS, resuspended in $1 \mathrm{mM}$ EDTA/PBS (pH 8.0) with $0.5 \% \mathrm{BSA}$, and passed through a cell strainer. Cells were analyzed on a Gallios flow cytometer (Beckman-Coulter, Inc.) and data were analyzed using FlowJo v10 software (FlowJo LLC).

ER tracker staining. The ACHN cells (ACHN/sgControl, ACHN/sgELOVL2-1 and ACHN/sgELOVL2-2) were seeded on glass coverslips in $60-\mathrm{mm}$ dishes and cultured at $37^{\circ} \mathrm{C}$ for $48 \mathrm{~h}$ in a $5 \% \mathrm{CO}_{2}$ incubator. The medium was then aspirated, and the cells were washed twice with HBSS before fixation with $4 \%$ formaldehyde (FUJIFILM Wako Pure Chemical Corporation) for $5 \mathrm{~min}$ at room temperature. The cells were then washed twice with HBSS and incubated with 500 nM ER Tracker Red (Thermo Fisher Scientific, Inc.; cat. no. E34250) for $2 \mathrm{~h}$ in the dark at $37^{\circ} \mathrm{C}$. Subsequently, the cells were washed twice with PBS and the coverslips were mounted on glass slides. Images of the stained cells were acquired with a BZ-X710 fluorescence microscope (Keyence Corporation).

Live cells were incubated with $500 \mathrm{nM}$ ER Tracker in HBSS for 30 min before washing twice in HBSS, re-suspended in $1 \mathrm{mM}$ EDTA/PBS (pH 8.0) with $0.5 \%$ BSA, and passed through a cell strainer. Cells were analyzed on a Gallios flow cytometer and data were analyzed using FlowJo v10 software.
Gas chromatography-mass spectrometry $(G C-M S)$. The extraction of total lipids from cell pellets was performed as previously described (27). The hydrolysis and derivatization of the extracts to FA methyl esters (FAMEs) was performed. For the internal standards of conjugated FAs, C20:4 ( $\omega-6)$,

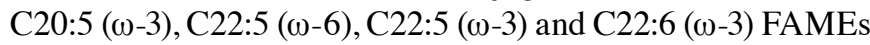
were purchased from MilliporeSigma or Cayman Chemical Company.

GC-MS analysis was performed using a GCMS-TQ8040 (Shimadzu Corporation) with an Omegawax ${ }^{\circledR}$ Capillary GC Column (MilliporeSigma; cat. no. 24136). The temperature gradient was initially increased from 70 to $150^{\circ} \mathrm{C}$ at a rate of $20^{\circ} \mathrm{C}$ per min and from 150 to $280^{\circ} \mathrm{C}$ at a rate of $8^{\circ} \mathrm{C}$ per min before decreasing from 280 to $200^{\circ} \mathrm{C}$ at a rate of $15^{\circ} \mathrm{C}$ per min. Sample injection was performed in splitless mode with $1.0 \mu \mathrm{l}$ of sample injected. For MS analysis, the ion source and interface temperature were set to $200^{\circ}$ and $270^{\circ} \mathrm{C}$, respectively. The data were acquired in full scan mode $(30-600 \mathrm{~m} / \mathrm{z})$ under $70 \mathrm{eV}$ of ionization voltage. FAMEs were identified according to retention time and electron ionization-MS (EI-MS) spectrum matching with reference FAME standards. The relative amount of FAMEs was calculated by comparing average peak area per sample mass. Each sample was independently measured three times.

Statistical analysis. Data are expressed as the mean \pm SD. All statistical analyses were performed using JMP 10 software (SAS Institute, Inc.), GraphPad Prism8 (GraphPad Software, Inc.) or R package (version 4.0.2; RStudio, Inc.). The significance of the differences between the two groups was assessed using the unpaired Student's t-test or Mann-Whitney test. The significance of the differences between the three or more groups was assessed using one-way ANOVA with post hoc comparisons using Dunnett's test or Kruskal-Wallis test followed by a Dunn's post hoc test with Bonferroni correction. Survival curves were constructed using the Kaplan-Meier method and the difference between the curves was evaluated using the log-rank test. Patients were divided into two groups, a low expression or high expression group, using the cutoff of median expression values. $\mathrm{P}<0.05$ was considered to indicate a statistically significant difference.

\section{Results}

ELOVL2 is highly overexpressed in RCC. The abundance of seven ELOVL isozymes in corresponding normal and tumor tissues was first examined in the present cohort, revealing that the ELOVL1, ELOVL2, ELOVL5 and ELOVL7 expression levels were elevated in ccRCC tissues (Fig. 1A). To validate these results, ELOVL isozyme gene expression in corresponding normal and tumor tissues was examined using TCGA database (KIRC cohort). It was confirmed that ELOVL2 mRNA expression was most highly and significantly elevated in ccRCC tissues (Fig. 1B; P<0.0001). Subsequently, ELOVL2 mRNA expression was investigated further among three major histological subtypes of RCC using TCGA database (KIRC, KIRP and KICH cohorts). This revealed that ELOVL2 was overexpressed in the pRCC and chRCC tissues; however, its expression in ccRCC tissues was the highest among the histological subtypes (Fig. 1C). Moreover, the ELOVL2 mRNA 


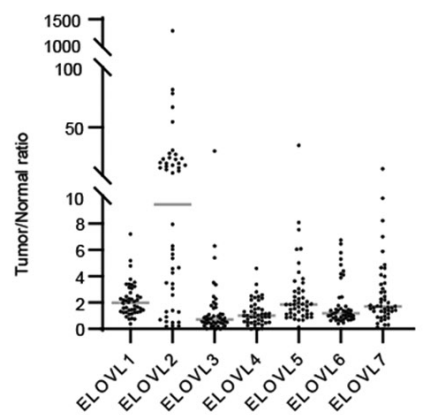

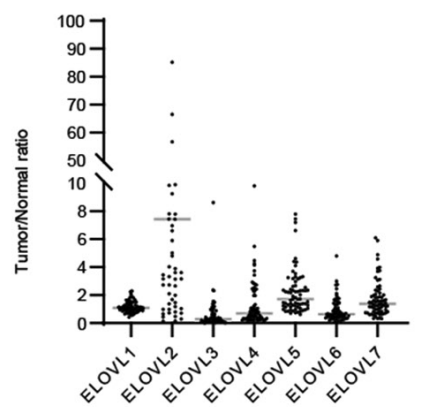

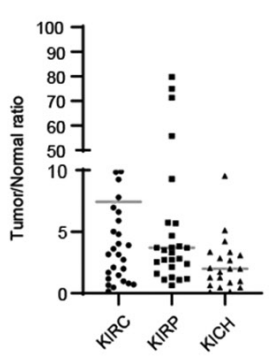

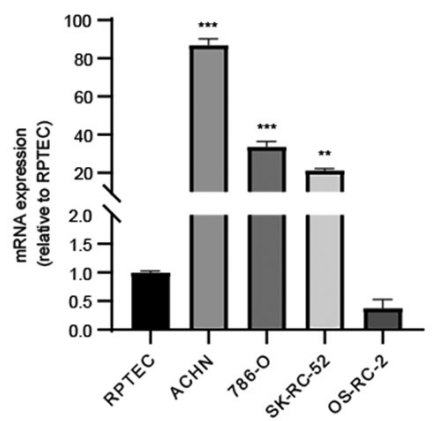

$\mathrm{E}$
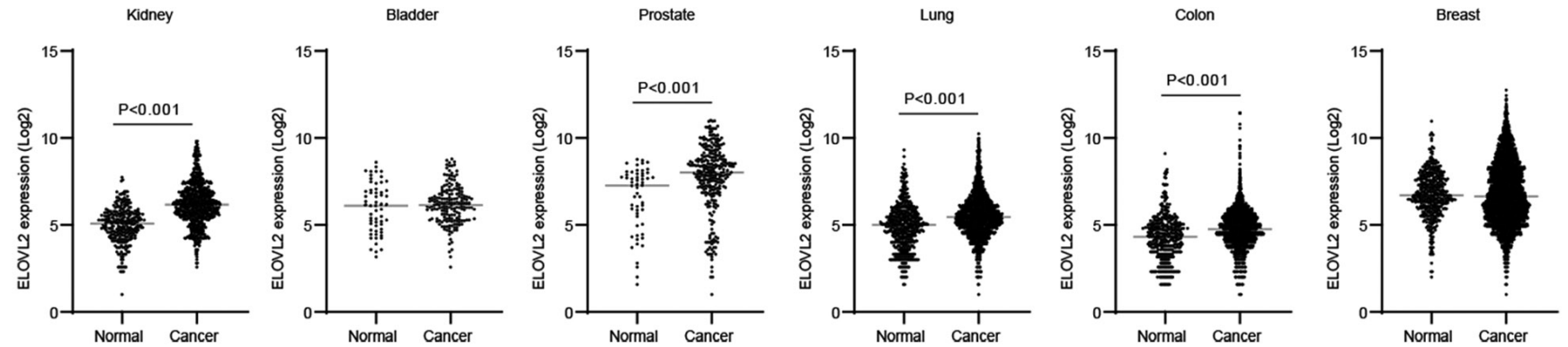

Figure 1. The abundance of seven ELOVL isozymes in RCC tissues and RCC cell lines. (A) ELOVL isozyme mRNA expression in the present cohort (n=46), (B) ELOVL isozyme mRNA expression in the TCGA KIRC cohort ( $\mathrm{n}=71)$. (C) ELOVL2 mRNA expression in the TCGA KIRC ( $\mathrm{n}=71)$, KIRP ( $\mathrm{n}=31)$ and KICH cohorts ( $n=23)$. (D) ELOVL2 mRNA expression in RCC cell lines. (C and D) P-values were assessed using ANOVA with post hoc comparisons using Dunnett's test. ${ }^{* *} \mathrm{P}<0.01$ and ${ }^{* * *} \mathrm{P}<0.001$, vs. RPTEC cells. (E) ELOVL2 gene expression patterns comparison between normal and tumor tissues in tumor samples. P-values were assessed using a Mann-Whitney test. ELOVL, very-long-chain fatty acid; RCC, renal cell carcinoma; TCGA, The Cancer Genome Atlas; KIRC; kidney renal clear cell carcinoma cohort; KIRP, cervical kidney renal papillary cell carcinoma cohort; KICH, kidney chromophobe cohort.

expression levels were markedly elevated in the ACHN, 786-O and SK-RC-52 cell lines, although the mRNA expression levels in OS-RC-2 cell line were lower as compared with those in the RPTEC cell line (Fig. 1D).

Subsequently, the cancer-associated alteration of ELOVL2 gene expression in various types of cancer was investigated using the GENT2 database (Fig. 1E). Compared with normal tissues, ELOVL2 expression was found to be significantly higher in kidney, prostate, lung and colon cancers, whereas ELOVL2 expression was similar in bladder or breast cancers. These results suggested that the roles of ELOVL isozymes differed by cancer type and that ELOVL2 overexpression may be associated with the carcinogenesis or disease progression of RCC, particularly ccRCC.

ELOVL2 overexpression is associated with the progression of $R C C$. The association between the gene expression of ELOVL2 and tumor-node-metastasis (TNM) stages in the KIRC cohort was examined, in order to clarify the clinical significance of ELOVL2 in ccRCC. ELOVL2 expression was higher in locally advanced and metastatic disease, although its expression was not associated with the lymph node metastasis status (Fig. 2A-C). Collectively, ELOVL2 expression was increased according to clinical stage (Fig. 2D).

Subsequently, the association between the expression of ELOVL2 and the prognosis of patients with RCC was evaluated, in order to elucidate the clinical impact of ccRCC. According to Kaplan-Meier analysis, it was revealed that the high expression of ELOVL2 was significantly associated with a poor prognosis in the present cohort $(\mathrm{P}=0.0015$, Fig. 2E). To validate these results, the association between the expression of
ELOVL2 and the prognosis of patients with ccRCC was examined further in the KIRC cohort and it was demonstrated that, similarly, the high expression of ELOVL2 was significantly associated with a poor prognosis ( $\mathrm{P}<0,01, \mathrm{Fig} .2 \mathrm{~F})$. Moreover, a high expression of ELOVL2 was significantly associated with a poor prognosis of patients with pRCC ( $\mathrm{P}<0.0001$, Fig. $2 \mathrm{G})$. However, this was not observed in patients with chRCC (Fig. 2H). In total, the aforementioned results indicated that ELOVL2 may mediate ccRCC and pRCC disease progression, at least partially.

ELOVL2 promotes the proliferation of renal cancer cells. To assess the function of ELOVL2 in the proliferation of RCC cells, the shRNA knockdown of ELOVL2 in 786-O, ACHN and SK-RC-52 cells was performed. The effects of each shRNA were evaluated using RT-qPCR and a significant decrease in ELOVL2 expression was confirmed (Fig. 3A). MTT assays were then performed to examine the effects of ELOVL2 knockdown on cellular proliferation. It was observed that the knockdown of ELOVL2 inhibited the proliferation of all cells compared with the cells transfected with control shRNA (Fig. 3B).

By contrast, ELOVL2 overexpression was induced in the OS-RC-2 cells, a cell line with a lower basal expression of ELOVL2. The transfection efficacy was evaluated using RT-qPCR and significantly increased expression of ELOVL2 was confirmed (Fig. 3C). MTT assays revealed that the proliferation of ELOVL2-overexpressing cells was significantly promoted, in comparison with the control group (Fig. 3D), indicating that ELOVL2 may be a promoter of the proliferation of renal cancer cells. 
A

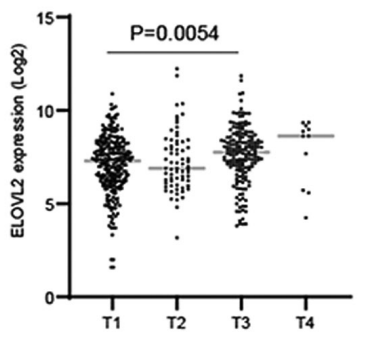

E

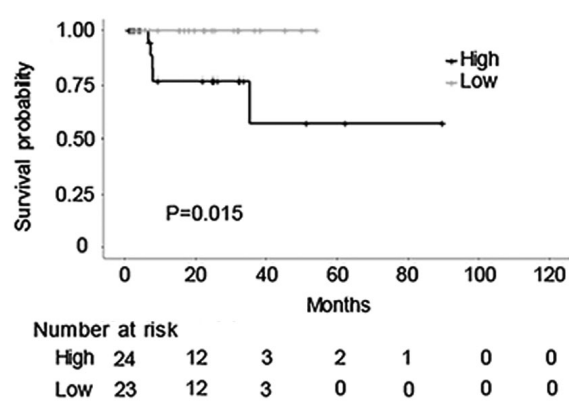

G

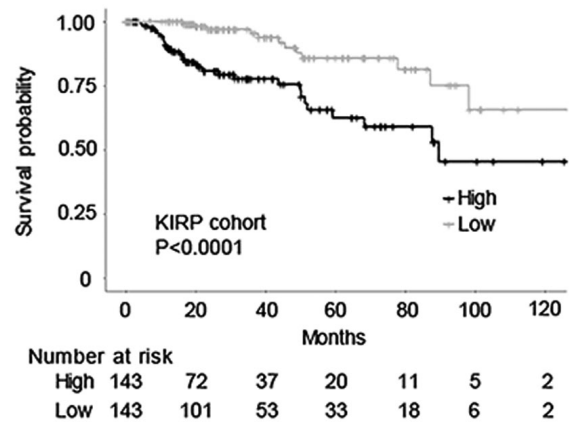

C

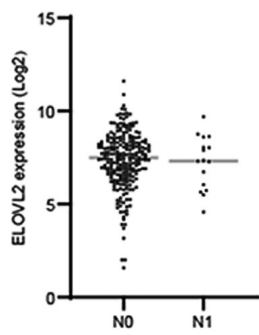

D

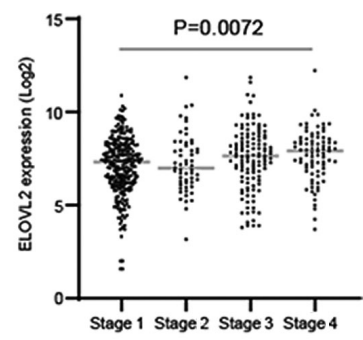

F

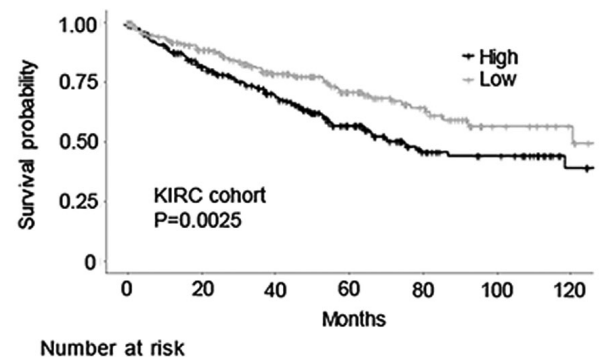

$\begin{array}{lllllll}\text { High } 265 & 189 & 135 & 75 & 35 & 25 & 8 \\ \text { Low } 265 & 193 & 127 & 73 & 41 & 14 & 8\end{array}$

$\mathrm{H}$

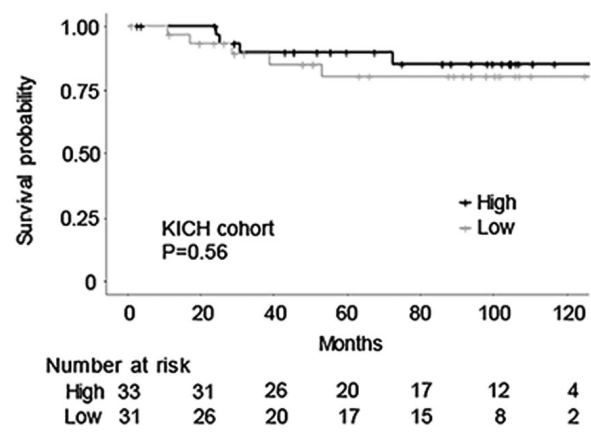

Figure 2. Association between ELOVL2 gene expression and clinical significance in patients with RCC. (A) The association between the mRNA expression of ELOVL2 isozymes and T stage in the TCGA KIRC cohort $(\mathrm{n}=530)$. (B) Association between the mRNA expression of ELOVL2 isozymes and N stage in the TCGA cohort $(\mathrm{n}=256)$. N stage data not available in 274 patients. (C) Association between the mRNA expression of ELOVL2 isozymes and M stage in the TCGA cohort $(n=498)$. M stage data not available in 32 patients. (D) Association between ELOVL2 isozyme mRNA expression and clinical stage in the TCGA cohort (n=527). Clinical stage data were not available for 3 patients. (E) Kaplan-Meier curves of the overall survival of 46 ccRCC patients in the present cohort with respect to the ELOVL2 mRNA level. (F) Kaplan-Meier curves of the overall survival of 530 ccRCC patients in the TCGA KIRC cohort with respect to ELOVL2 mRNA levels. (G) Kaplan-Meier curves of the overall survival of 286 pRCC patients in the TCGA KIRP cohort with respect to ELOVL2 mRNA levels. (H) Kaplan-Meier curves of the overall survival of 64 chRCC patients in the TCGA KICH cohort with respect to ELOVL2 mRNA levels. P-values were assessed using a Mann-Whitney test (B and C), or the Kruskal-Wallis test followed by a Dunn's post hoc test with Bonferroni correction (A and D) or the log-rank test (E-H). ELOVL, very-long-chain fatty acid; TCGA, The Cancer Genome Atlas; KIRC; kidney renal clear cell carcinoma; KIRP, cervical kidney renal papillary cell carcinoma; KICH, kidney chromophobe cohort ccRCC, clear cell RCC; pRCC, papillary PCC; chRCC, chromophobe RCC.

ELOVL2 ablation suppresses tumor growth in vivo, the synthesis of PUFAs and the production of LDs in renal cancer cells. To further clarify the roles of ELOVL2 in RCC progression, an ELOVL2-knockout ACHN cell line was established, by using a CRISPR/Cas9 system (sgELOVL2-1 and sgELOVL2-2) (Fig. S1). In vitro, cellular proliferation was significantly decreased by ELVOVL2 ablation in the ACHN cells (Fig. S1). More importantly, the CRISPR/Cas9-mediated ablation of ELOVL2 suppressed tumor growth when the cells were implanted subcutaneously into mice (Fig. 4A). The maximum volumes of the subcutaneous xenograft tumors in the ACHN/sgControl and ACHN/sgELOVL2-2 at the day of sacrifice were 241 and $109 \mathrm{~mm}^{3}$, respectively. Of note, all xenograft tumors in the ACHN/sgELOVL2-1 group spontaneously regressed at 14 days following transplantation and were not detected at the time of extirpation. The Ki-67 index was also examined in the xenograft tumors transfected with sgControl or sgELOVL2 and it was observed that the ACHN/sgELOVL2-2 cells exhibited a significantly lower Ki-67 index than the ACHN/sgControl cells (Fig. 4B and C). These results further supported the possibility that ELOVL2 augments tumor growth in vivo.

As ELOVL2 is located on chromosome 6p24.2 and encodes for an endoplasmic reticulum transmembrane protein controlling the elongation of C20-C24 PUFAs (7), total FA levels were then evaluated in the ACHN/sgControl and ACHN/sgELOVL2 cells using GC-MS analysis (Fig. S2). The alteration of LC-PUFA levels is demonstrated in Fig. 4D. ELOVL2 is an essential enzyme in the endogenous production of docosahexaenoic acid (DHA, C22:6 n-3) $(28,29)$ and, as expected, the DHA levels were significantly decreased by the ablation of ELOVL2 in the renal cancer cells. Moreover, the 
A

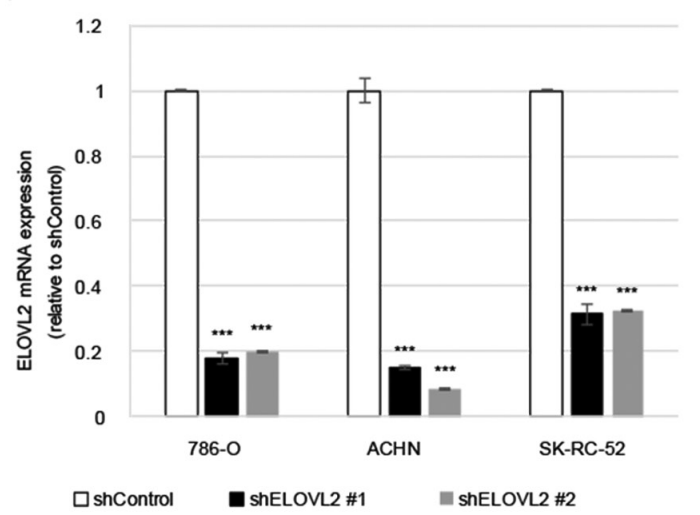

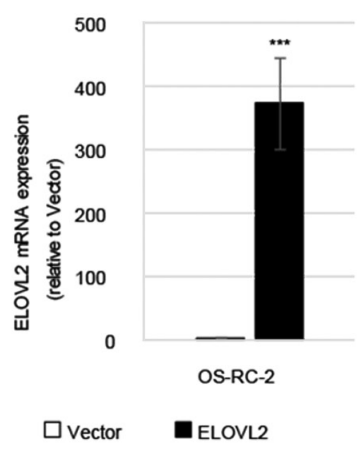

D

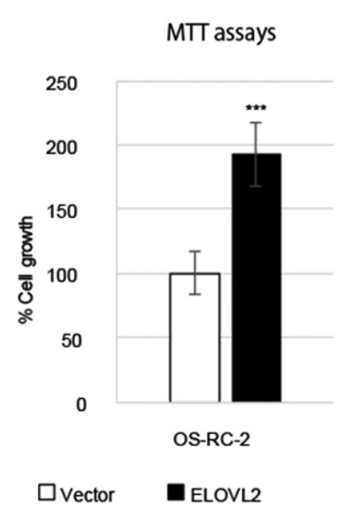

Figure 3. ELOVL2 promotes cellular proliferation in renal cancer cells in vitro. (A) ELOVL2 mRNA expression in renal cancer cells infected with scramble or ELOVL2 shRNA. (B) Analysis of cell proliferation by using MTT assay in renal cancer cells infected with scramble or ELOVL2 shRNA after 72 h. (C) The mRNA expression of ELOVL2 in OSRC-2 cells transfected with control or ELOVL2 overexpression vectors. (D) Analysis of cellular proliferation by MTT assay in OSRC-2 cells transfected with control or ELOVL2 overexpression vectors after 120 h. P-values were assessed using ANOVA with post hoc comparisons using Dunnett's test (A and B) or the Student's t-test (C and D). ${ }^{* *} \mathrm{P}<0.01$ and ${ }^{* * * *} \mathrm{P}<0.001$, vs. control. ELOVL, very-long-chain fatty acid.

amounts of other LC-PUFAs species, including arachidonic acid (AA, C20:4 n-6), eicosapentaenoic acid (EPA) and docosapentaenoic acid (DPA), were also significantly decreased. On the other hand, the production of DPA was not consistently altered in the ACHN/sgELOVL2 cells. The amount of DPA in the ACHN cells was very low and was not detected in several samples (Fig. S2).

Since previous studies have demonstrated that ELOVL2 promotes the accumulation of LDs through the production of DHA $(11,12)$, the storage of LDs was further assessed using neutral lipid staining. Of note, the abundance of LDs in the ACHN/sgELOVL 2 cells was significantly decreased compared with the ACHN/sgControl cells (Fig. 4E-4G), suggesting that the alteration of lipid metabolism by ELOVL2 overexpression may affect RCC cell proliferation.

ELOVL2 ablation induces the apoptosis of renal cancer cells. Previous studies have revealed that the production of intracellular LDs promotes apoptosis under stressful tumor microenvironments (4). Therefore, in the present study, apoptosis was evaluated in ACHN/sgControl or ACHN/sgELOVL2 cells and a significantly elevated activity of caspase $3 / 7$ was detected in the ACHN/sgELOVL2 cells (Fig. 5A). Similarly, a greater number of apoptotic ACHN/sgELOVL2 cells compared with ACHN/sgControl cells was identified, as evidenced by Annexin V/PI staining (Fig. 5B). Depending on cellular stress, intrinsic apoptosis (30) leads to the loss of mitochondrial membrane potential; thus, the present study further assessed the mitochondrial transmembrane electric potential of $\mathrm{ACHN} / \mathrm{sgControl}$ or ACHN/sgELOVL2 cells using fluorescent JC-1. The aggregate/monomer ratio was significantly decreased in the ACHN/sgELOVL2 cells (Fig. 5C), indicating the promotion of mitochondrial transmembrane polarization and the activation of the intrinsic apoptotic pathway by ELOVL2 ablation. More precisely, pro-apoptotic gene (BAX, BAK, PUMA and NOXA) expression levels were significantly increased, while anti-apoptotic gene (BCL2 and MCL1) expression levels were significantly decreased due to ELOVL2 ablation (Fig. 5D). These results indicated that ELOVL2 contributes to cellular survival through the inhibition of theapoptosis of renal cancer cells.

The degradation of lipid storage capacity in LD form can lead to the collapse of ER homeostasis, triggering the unfolded protein response (UPR) and cellular apoptosis $(4,5)$. Therefore, in order to support the hypothesis of the involvement of ELOVL2 expression in ER homeostasis, ER tracker staining was performed in ACHN/sgControl or ACHN/sgELOVL2 cells. Subsequent ER imaging (Fig. 5E) and quantification using flow cytometry (Fig. 5F and G) indicated ER expansion in the ACHN/sgELOVL2 cells, due to ER stress. Additionally, ELOVL2 ablation promoted the phosphorylation of IRE1 $\alpha$, an activator of UPR sensors (Fig. 5H), while the expression of 


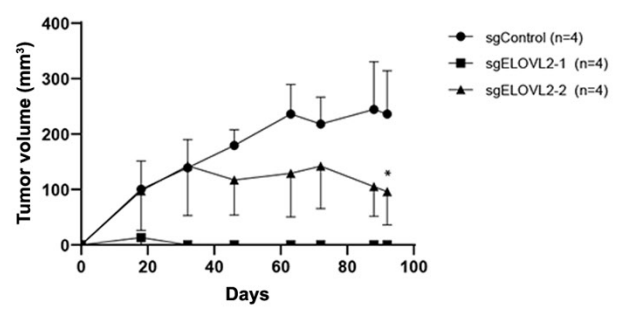

D
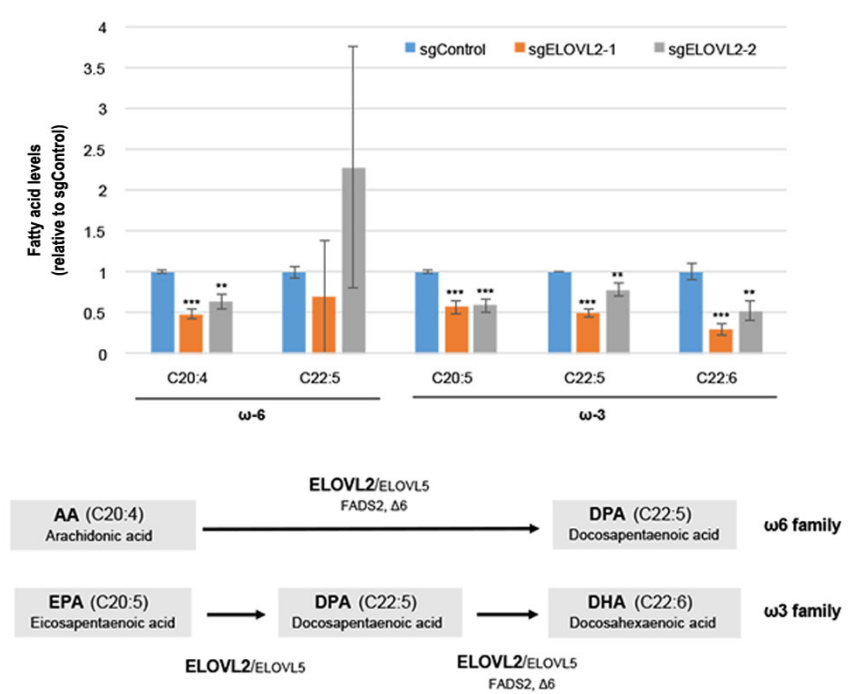
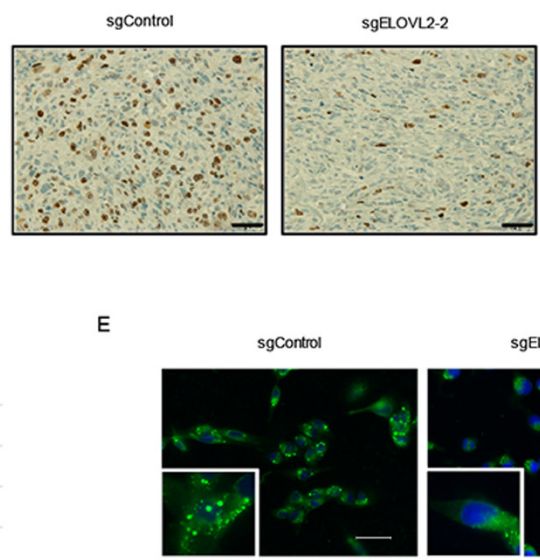

$\mathrm{F}$
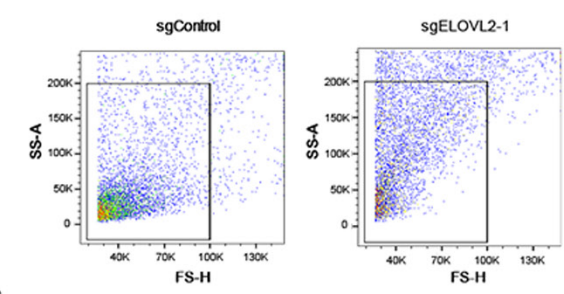

sgElOV2-1
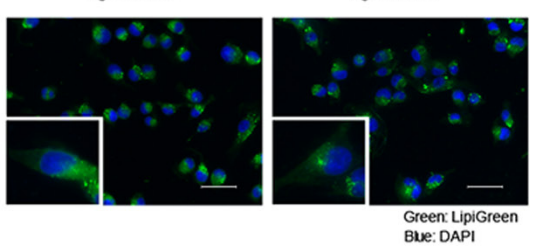

Green LipiG
Bue: DAPI

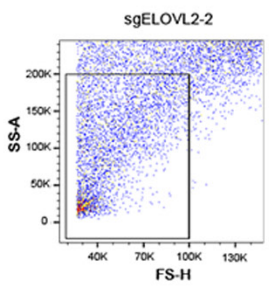

G

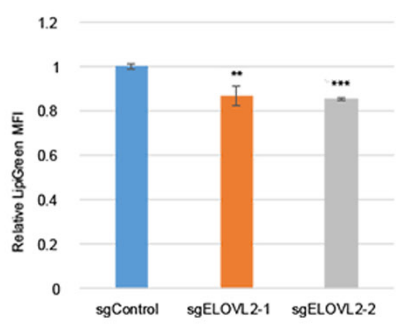

Figure 4. CRISPR/Cas9-mediated knockdown of ELOVL2 inhibits tumor growth in vivo and alters lipid metabolism of ACHN cells. (A) Sequential changes of subcutaneous xenograft tumors from ACHN/sgControl or ACHN/sgELOVL2 cells. (B) Representative images of Ki-67 staining of xenograft tumors in ACHN/sgControl or ACHN/sgELOVL2-2 cells. Scale bar, $50 \mu \mathrm{m}$. (C) Ki-67 labeling index of xenograft tumors in ACHN/sgControl or ACHN/sgELOVL2-2 cells. (D) LC-PUFA measurement by GC-MS in ACHN/sgControl or ACHN/sgELOVL2 cells. (E) Representative images of lipid droplets in ACHN/sgControl or ACHN/sgELOVL2 cells. Scale bar $50 \mu \mathrm{m}$. (F) Representative flow cytometry dot plots of LipiGreen staining in ACHN/sgControl or ACHN/sgELOVL2 cells. (G) LipiGreen fluorescence quantification in ACHN/sgControl or ACHN/sgELOVL2 cells. P-values were assessed using the Mann-Whitney test (A and $\mathrm{C}$ ) or ANOVA with post hoc comparisons using Dunnett's test (D and G). ${ }^{*} \mathrm{P}<0.05,{ }^{* * *} \mathrm{P}<0.01$ and ${ }^{* * * *} \mathrm{P}<0.001$, vs. control. ELOVL, very-long-chain fatty acid; sg, single-guided; LC-PUFA, long-chain polyunsaturated fatty acids; GC-MS, gas chromatography/mass spectrometry.

CHOP, which plays a main role in ER stress-induced apoptosis, was upregulated by ELOVL2 ablation (Fig. 5H). Collectively, these results demonstrated that ELOVL2 mediated-lipid metabolism suppresses cell apoptosis in renal cancer cells and suggested that disruption of ER homeostasis may be a potential mechanism of induction.

\section{Discussion}

The present study demonstrated that ELVOL2 may alter lipid metabolism and promote tumor growth via the inhibition of the apoptosis of renal cancer cells. Moreover, it was observed that ELOVL2 expression in RCC tissues was elevated and that the higher expression of ELOVL2 was significantly associated with a poor prognosis of patients with RCC.

There are limited studies available on the roles of ELOVL2 in cancer progression $(22,31,32)$ and their results are controversial. Gimple et al (22) demonstrated that ELOVL2 promoted
LC-PUFA synthesis, supporting efficient EGFR signaling and the proliferation of glioblastoma stem cells. By contrast, Kang et al (31) reported that ELOVL2 ablation may promote cell migration and the colony formation of breast cancer cells and revealed that a decreased ELOVL2 expression was associated with a poorer prognosis of patients with breast cancer. Furthermore, Ding et al (32) reported that ELOVL2 suppresses the proliferation of neuroblastoma cells and was associated with favorable survival rates. According to the conflicting findings reported in previous studies, a multi-role function for ELOVL2 in cancer progression has been demonstrated, that clearly varies by cancer type.

An altered lipid metabolism greatly affects cancer progression, an effect observed in the results of the present study, being in line with research depicting the role of ELOVL2 as a primary controller of the elongation process of LC-PUFAs and a critical enzyme in DHA biosynthesis (7). In the present study, it was demonstrated that endogenous LC-PUFAs, 


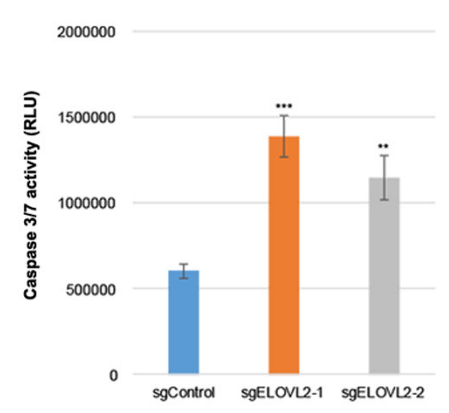

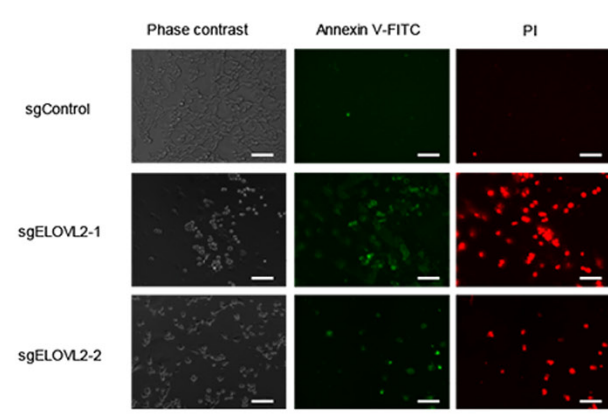

E
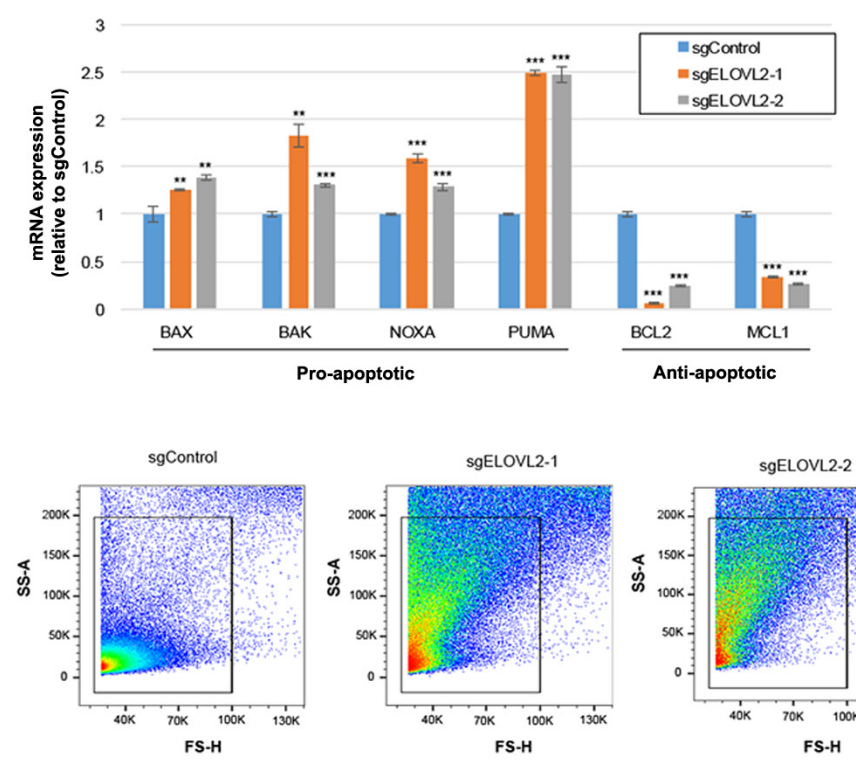

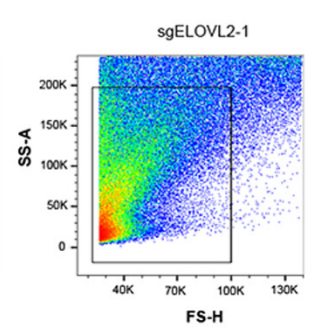

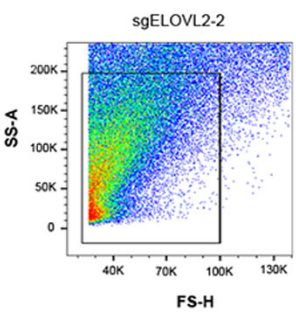

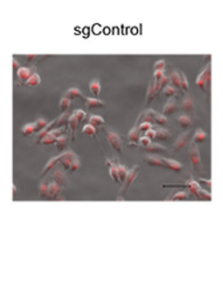

c

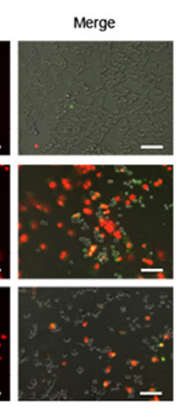

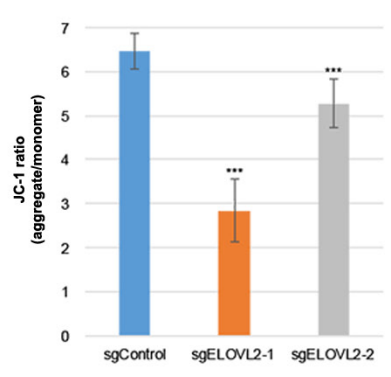

Figure 5. CRISPR/Cas9-mediated knockdown of ELOVL2 promotes the loss of mitochondrial transmembrane potential and apoptosis in ACHN cells (A) Analysis of cell apoptosis by caspase 3/7 assay in ACHN/sgControl or ACHN/sgELOVL2 cells. (B) Representative images of Annexin V-FITC staining in ACHN/sgControl or ACHN/sgELOVL2 cells. Scale bar, $100 \mu \mathrm{m}$. (C) Aggregates/monomers relative ratio evaluation in ACHN/sgControl or ACHN/sgELOVL2 cells by using JC-1 assay. (D) Apoptosis-related gene expression levels in ACHN/sgControl or ACHN/sgELOVL2 cells. (E) Representative images of ER tracker staining in ACHN/sgControl or ACHN/sgELOVL2 cells. Scale bar, $50 \mu \mathrm{m}$. (F) Representative flow cytometry dot plots of ER tracker staining in ACHN/sgControl or ACHN/sgELOVL2 cells. (G) ER tracker fluorescence quantification in ACHN/sgControl or ACHN/sgELOVL2 cells. (H) Western blot analysis of pIRE1 $\alpha$, IRE1 $\alpha$, and CHOP in ACHN/sgControl or ACHN/sgELOVL2 cells. P-values were assessed using ANOVA with post hoc comparisons using Dunnett's test (A, C, D and G). ${ }^{* *} \mathrm{P}<0.01$ and ${ }^{* * *} \mathrm{P}<0.001$, vs. control. ELOVL, very-long-chain fatty acid; sg, single guided; IRE1 $\alpha$, serine/threonine-protein kinase/endoribonuclease inositol-requiring enzyme $1 \alpha$; pIRE1 $\alpha$, phosphorylated-IRE1 $\alpha$; CHOP, C/EBP homologous protein.

including AA and DHA, were decreased due to ELOVL2 ablation in renal cancer cells. In relation to this, it has been previously reported that the inhibition of the AA pathway by lipoxygenase (LOX) inhibitors induces the apoptosis and reduces the viability of renal cancer cells in vitro $(33,34)$. Although the ELOVL2 overexpression results of the present study are in line with these mechanistic findings, it has been previously reported, however, that DHA administration may inhibit the proliferation and invasion of renal cancer cells in vitro $(35,36)$. However, LC-PUFAs are known to have distinct and contrasting effects on cancer progression. Therefore, the alteration of a delicate balance among a variety of LC-PUFAs, due to the inhibition of ELOVL2 may be associated with the differing phenotypes observed in various cancer types.

Previous studies have demonstrated that LDs are increased through the endogenous production of DHA by ELOVL2 in mice $(11,12)$. Similarly, it was revealed that ELOVL2 ablation may suppress the production of DHA and LDs in renal cancer cells, suggesting that ELOVL2 overexpression may promote LD production through endogenous DHA production in RCC. Cancer cells are often found in harsher environmental (macro and micro) conditions, including hypoxia and nutrient deprivation, yet still rapidly grow under such severe stressors. The functions of LDs under conditions of cellular stress, such as the maintenance of energy and redox homeostasis, the regulation of autophagy, the maintenance of ER homeostasis and protection against lipotoxicity, have been demonstrated (4).

Ackerman et al (6) revealed that LDs prevent the accumulation of toxic, saturated lipids during hypoxia by buffering of cellular lipid saturation through exchange of TG-resident unsaturated FAs in ccRCC. Cellular lipotoxicity due to saturated FAs (SFA), including palmitate (C16:0), may cause apoptosis, leading to cancer cell death $(37,38)$. Recently, ELOVL2 was designated as a critical pro-survival enzyme, assisting in the prevention of glucolipotoxicity-induced $\beta$-cell apoptosis (39). Of note, ELOVL2/DHA axis-modified lipid partitioning results in a non-toxic utilization of SFA 
palmitate by favoring its transport into mitochondria for FA oxidation (FAO) through a CPT1-dependent mechanism. Additionally, Balaban et al demonstrated that $\mathrm{C} 4-2 \mathrm{~B}$ prostate cancer cells and MCF-7 breast cancer cells are protected from palmitate-induced apoptosis by mitochondrial FAO $(37,38)$. During palmitate-induced lipotoxicity, cellular levels of anti-apoptotic proteins, including BCL-2 or MCL-1, have been reported to be decreased $(40,41)$ while levels of pro-apoptotic proteins, including PUMA or NOXA, have been reported to be increased $(42,43)$. It was revealed in the present study that ELOVL2 ablation may promote renal cancer cell apoptosis through the alteration of pro- and anti-apoptotic genes in a similar manner. These findings suggest that ELOVL2 may protect cells against lipotoxicity-driven apoptosis to promote tumor growth in RCC.

Additionally, it was demonstrated that ELOVL2 ablation in RCC may promote ER stress and CHOP upregulation, downregulating BCL-2 and MCL-1, while upregulating BAK and BAX, via a mitochondria-dependent pathway (44). Indeed, the results of the present study demonstrated that pro- and anti-apoptotic genes were similarly altered by ELOVL2 ablation. In line with the findings in the study by Qui et al (5), who reported that HIF2 $\alpha /$ PLIN2-dependent LDs promote resistance against ER stress and cell survival in ccRCC, these collective findings are in support of ER stress promoting cellular apoptosis by ELOVL2 ablation in renal cancer cells, decreasing LDs and removing the cellular buffer against toxic lipids.

In conclusion, the present study demonstrated for the first time, to the best of our knowledge, that ELOVL2 promotes tumor growth by the inhibition of apoptosis via the elongation of PUFAs, at least partly by maintaining ER homeostasis in renal cancer cells. Taken together, it was suggested that ELOVL2-induced LDs may contribute to cancer progression due to multiple protective effects against cellular stress in RCC. Moreover, it was suggested that ELOVL2 may be an attractive potential therapeutic target for patients with RCC.

\section{Acknowledgements}

The authors are grateful for the skillful technical assistance of Mr. Minoru Suzuki, Mrs. Naoko Ueki, Mrs. Noriko Kunita (University of Tsukuba).

\section{Funding}

The present study was supported by the Japan Society for the Promotion of Science KAKENHI grant (nos. 17K16774 and 19K09664) and the COI-NEXT grant (no. JPMJPF2017).

\section{Availability of data and materials}

The datasets used and/or analyzed during the current study are available from the corresponding author on reasonable request.

\section{Authors' contributions}

SK, TK and TMa contributed to the conceptualization of the present study. HNi, HS and TAS contributed to the design of the present study. SS, KeT, KoT, MS, YN, SN, MW and
TMi contributed to performing the experiments and the data analyses. KeT, SK,YN and BJM wrote the original draft. KeT, SK, TK, HNe, BJM and TS contributed to the interpretation of the results in the present study. KeT and SK confirmed the authenticity of all the raw data. All authors have read and approved the final manuscript.

\section{Ethics approval and consent to participate}

All protocols have been approved by the Ethics Committee of the University of Tsukuba (approval no. H28-104). All patients provided written informed consent prior to undergoing surgery. All animal studies were approved by the Animal Experiment Committee of the University of Tsukuba and all experiments were performed in accordance with the guidelines of the University of Tsukuba's Regulations of Animal Experiments (approval no. 20-370).

\section{Patient consent for publication}

Not applicable.

\section{Competing interests}

The authors declare that they have no competing interests.

\section{References}

1. Hsieh JJ, Purdue MP, Signoretti S, Swanton C, Albiges L, Schmidinger M, Heng DY, Larkin J and Ficarra V: Renal cell carcinoma. Nat Rev Dis Primers 3: 17009, 2017.

2. Rini BI, Battle D, Figlin RA, George DJ, Hammers H, Hutson T, Jonasch E, Joseph RW, McDermott DF, Motzer RJ, et al: The society for immunotherapy of cancer consensus statement on immunotherapy for the treatment of advanced renal cell carcinoma (RCC). J Immunother Cancer 7: 354, 2019.

3. Walther TC and Farese RV Jr: Lipid droplets and cellular lipid metabolism. Annu Rev Biochem 81: 687-714, 2012.

4. Petan T, Jarc E and Jusović M: Lipid Droplets in Cancer: Guardians of Fat in a Stressful World. Molecules 23: 1941, 2018.

5. Qiu B, Ackerman D, Sanchez DJ, Li B, Ochocki JD, Grazioli A, Bobrovnikova-Marjon E, Diehl JA, Keith B and Simon MC: HIF $2 \alpha$-Dependent Lipid Storage Promotes Endoplasmic Reticulum Homeostasis in Clear-Cell Renal Cell Carcinoma. Cancer Discov 5: 652-667, 2015.

6. Ackerman D, Tumanov S, Qiu B, Michalopoulou E, Spata M, Azzam A, Xie H, Simon MC and Kamphorst JJ: Triglycerides promote lipid homeostasis during hypoxic stress by balancing fatty acid saturation. Cell Rep 24: 2596-2605.e5, 2018.

7. Guillou H, Zadravec D, Martin PG and Jacobsson A: The key roles of elongases and desaturases in mammalian fatty acid metabolism: Insights from transgenic mice. Prog Lipid Res 49: 186-199, 2010.

8. von Roemeling CA, Marlow LA, Wei JJ, Cooper SJ, Caulfield TR, Wu K, Tan WW, Tun HW and Copland JA: Stearoyl-CoA desaturase 1 is a novel molecular therapeutic target for clear cell renal cell carcinoma. Clin Cancer Res 19: 2368-2380, 2013.

9. Lucarelli G, Ferro M, Loizzo D, Bianchi C, Terracciano D, Cantiello F, Bell LN, Battaglia S, Porta C, Gernone A, et al: Integration of Lipidomics and Transcriptomics Reveals Reprogramming of the Lipid Metabolism and Composition in Clear Cell Renal Cell Carcinoma. Metabolites 10: 509, 2020.

10. Yang H, Zhang X, Liu F, Fan J, Wang B and Dong C: SREBP1-driven lipid desaturation supports clear cell renal cell carcinoma growth through regulation of NF- $\kappa B$ signaling. Biochem Biophys Res Commun 495: 1383-1388, 2018.

11. Pauter AM, Olsson P, Asadi A, Herslöf B, Csikasz RI, Zadravec D and Jacobsson A: Elovl2 ablation demonstrates that systemic DHA is endogenously produced and is essential for lipid homeostasis in mice. J Lipid Res 55: 718-728, 2014. 
12. Kobayashi T, Zadravec D and Jacobsson A: ELOVL2 overexpression enhances triacylglycerol synthesis in 3T3-L1 and F442A cells. FEBS Lett 581: 3157-3163, 2007.

13. $\operatorname{Lim~K}$, Han $\mathrm{C}, \mathrm{Xu} \mathrm{L}$, Isse $\mathrm{K}$, Demetris AJ and $\mathrm{Wu} \mathrm{T}$ : Cyclooxygenase-2-derived prostaglandin E2 activates beta-catenin in human cholangiocarcinoma cells: Evidence for inhibition of these signaling pathways by omega 3 polyunsaturated fatty acids. Cancer Res 68: 553-560, 2008.

14. Yao QH, Zhang XC, Fu T, Gu JZ, Wang L, Wang Y, Lai YB, Wang YQ and Guo Y: $\omega-3$ polyunsaturated fatty acids inhibit the proliferation of the lung adenocarcinoma cell line A549 in vitro. Mol Med Rep 9: 401-406, 2014.

15. Serhan CN, Hong S, Gronert K, Colgan SP, Devchand PR, Mirick G and Moussignac RL: Resolvins: A family of bioactive products of omega-3 fatty acid transformation circuits initiated by aspirin treatment that counter proinflammation signals. J Exp Med 196: 1025-1037, 2002.

16. Sobin LH, Gospodarowicz MK and Wittekind CH (eds): TNM Classification of Malignant Tumors. 7th edition. Wiley-Blackwell, Chichester, West Sussex, 2009.

17. Fuhrman SA, Lasky LC and Limas C: Prognostic significance of morphologic parameters in renal cell carcinoma. Am J Surg Pathol 6: 655-663, 1982.

18. Livak KJ and Schmittgen TD: Analysis of relative gene expression data using real-time quantitative PCR and the 2(-Delta Delta C(T)) Method. Methods 25: 402-408, 2001.

19. Kandori S, Kojima T, Matsuoka T, Yoshino T, Sugiyama A, Nakamura E, Shimazui T, Funakoshi Y, Kanaho Y and Nishiyama H: Phospholipase D2 promotes disease progression of renal cell carcinoma through the induction of angiogenin. Cancer Sci 109: 1865-1875, 2018

20. Grossman RL, Heath AP, Ferretti V, Varmus HE, Lowy DR, Kibbe WA and Staudt LM: Toward a Shared Vision for Cancer Genomic Data. N Engl J Med 375: 1109-1112, 2016.

21. Park SJ, Yoon BH, Kim SK and Kim SY: GENT2: An updated gene expression database for normal and tumor tissues. BMC Med Genomics 12 (Suppl 5): 101, 2019.

22. Gimple RC, Kidwell RL, Kim LJY, Sun T, Gromovsky AD, Wu Q, Wolf M, Lv D, Bhargava S, Jiang L, et al: Glioma Stem Cell-Specific Superenhancer Promotes Polyunsaturated Fatty-Acid Synthesis to Support EGFR Signaling. Cancer Discov 9: 1248-1267, 2019.

23. Purdy JG, Shenk T and Rabinowitz JD: Fatty acid elongase 7 catalyzes lipidome remodeling essential for human cytomegalovirus replication. Cell Rep 10: 1375-1385, 2015.

24. Cong L, Ran FA, Cox D, Lin S, Barretto R, Habib N, Hsu PD, Wu X, Jiang W, Marraffini LA, et al: Multiplex genome engineering using CRISPR/Cas systems. Science 339: 819-823, 2013

25. Miyamoto T, Lo PHY, Saichi N, Ueda K, Hirata M, Tanikawa C and Matsuda K: Argininosuccinate synthase 1 is an intrinsic Akt repressor transactivated by p53. Sci Adv 3: e1603204, 2017.

26. Mishra A,Zennami K, VelardeE,Thorek DLJ, Yegnasubramanian S, DeWeese TL and Lupold SE: Longitudinal measurement of subcutaneous and intratibial human prostate cancer xenograft growth and response to ionizing radiation by plasma Alu and LINE-1 ctDNA: A comparison to standard methods. Prostate 81: 745-753, 2021.

27. Folch J, Lees M and Sloane Stanley GH: A simple method for the isolation and purification of total lipides from animal tissues. J Biol Chem 226: 497-509, 1957.

28. Gregory MK and James MJ: Rainbow trout (Oncorhynchusmykiss) Elovl5 and Elovl2 differ in selectivity for elongation of omega-3 docosapentaenoic acid. Biochim Biophys Acta 1656-60: 2014, 1841.

29. Gregory MK and James MJ: Functional characterization of the duck and turkey fatty acyl elongase enzymes ELOVL5 and ELOVL2. J Nutr 144: 1234-1239, 2014.
30. Matsuura K, Canfield K, Feng W and Kurokawa M: Metabolic Regulation of Apoptosis in Cancer. Int Rev Cell Mol Biol 327: 43-87, 2016.

31. Kang YP, Yoon JH, Long NP, Koo GB, Noh HJ, Oh SJ, Lee SB, Kim HM, Hong JY, Lee WJ, et al: Spheroid-Induced Epithelial-Mesenchymal Transition Provokes Global Alterations of Breast Cancer Lipidome: A Multi-Layered Omics Analysis. Front Oncol 9: 145, 2019.

32. Ding Y, Yang J, Ma Y, Yao T, Chen X, Ge S, Wang L and Fan X: MYCN and PRC1 cooperatively repress docosahexaenoic acid synthesis in neuroblastoma via ELOVL2. J Exp Clin Cancer Res 38: 498, 2019.

33. Matsuyama M, Yoshimura R, Mitsuhashi M, Tsuchida K, Takemoto Y,Kawahito Y, Sano H and Nakatani T: 5-Lipoxygenase inhibitors attenuate growth of human renal cell carcinoma and induce apoptosis through arachidonic acid pathway. Oncol Rep 14: 73-79, 2005.

34. Matsuyama M and Yoshimura R: Relationship between arachidonic acid pathway and human renal cell carcinoma. OncoTargets Ther 1: 41-48, 2008.

35. Tasaki S, Horiguchi A, Asano T, Ito K, Asano T and Asakura H: Docosahexaenoic acid inhibits the phosphorylation of STAT3 and the growth and invasion of renal cancer cells. Exp Ther Med 14: 1146-1152, 2017

36. McCabe AJ, Wallace JMW, Gilmore WS, McGlynn H and Strain SJ: Docosahexaenoic acid reduces in vitro invasion of renal cell carcinoma by elevated levels of tissue inhibitor of metalloproteinase-1. J Nutr Biochem 16: 17-22, 2005.

37. Balaban S, Lee LS, Varney B, Aishah A, Gao Q, Shearer RF, Saunders DN, Grewal T and Hoy AJ: Heterogeneity of fatty acid metabolism in breast cancer cells underlies differential sensitivity to palmitate-induced apoptosis. Mol Oncol 12: 1623-1638, 2018.

38. Balaban S, Nassar ZD, Zhang AY, Hosseini-Beheshti E, Centenera MM, Schreuder M, Lin HM, Aishah A, Varney B, Liu-Fu F, et al: Extracellular Fatty Acids Are the Major Contributor to Lipid Synthesis in Prostate Cancer. Mol Cancer Res 17: 949-962, 2019.

39. Bellini L, Campana M, Rouch C, Chacinska M, Bugliani M, Meneyrol K, Hainault I, Lenoir V, Denom J, Véret J, et al: Protective role of the ELOVL2/docosahexaenoic acid axis in glucolipotoxicity-induced apoptosis in rodent beta cells and human islets. Diabetologia 61: 1780-1793, 2018.

40. Masuoka HC, Mott J, Bronk SF, Werneburg NW, Akazawa Y, Kaufmann SH and Gores GJ: Mcl-1 degradation during hepatocyte lipoapoptosis. J Biol Chem 284: 30039-30048, 2009.

41. Shimabukuro M, Wang MY, Zhou YT, Newgard CB and Unger RH: Protection against lipoapoptosis of beta cells through leptin-dependent maintenance of Bcl-2 expression. Proc Natl Acad Sci USA 95: 9558-9561, 1998.

42. Akazawa Y, Cazanave S, Mott JL, Elmi N, Bronk SF, Kohno S, Charlton MR and Gores GJ: Palmitoleate attenuates palmitate-induced Bim and PUMA up-regulation and hepatocyte lipoapoptosis. J Hepatol 52: 586-593, 2010.

43. Cazanave SC, Wang $X$, Zhou H, Rahmani M, Grant S, Durrant DE, Klaassen CD, Yamamoto $M$ and Sanyal AJ: Degradation of Keap1 activates BH3-only proteins Bim and PUMA during hepatocyte lipoapoptosis. Cell Death Differ 21: 1303-1312, 2014

44. Hu H, Tian M, Ding C and Yu S: The C/EBP Homologous Protein (CHOP) Transcription Factor Functions in Endoplasmic Reticulum Stress-Induced Apoptosis and Microbial Infection. Front Immunol 9: 3083, 2019.

This work is licensed under a Creative Commons Attribution-NonCommercial-NoDerivatives 4.0 International (CC BY-NC-ND 4.0) License. 